\title{
Accretion and diffusion in the DAZ white dwarf GALEX J1931+0117
}

\author{
Stéphane Vennes, Adéla Kawka and Péter Németh \\ Astronomický ústav, Akademie věd České republiky, Fričova 298, CZ-251 65 Ondřejov, Czech \\ Republic
}

\begin{abstract}
We present an analysis of high-dispersion and high signal-to-noise ratio spectra of the DAZ white dwarf GALEX J1931+0117. The spectra obtained with the VLT-Kueyen/UV-Visual Echelle Spectrograph show several well-resolved Si II spectral lines enabling a study of pressure effects on line profiles. We observed large Stark shifts in silicon lines in agreement with laboratory measurements. A model atmosphere analysis shows that the magnesium, silicon and iron abundances exceed solar abundances, while the oxygen and calcium abundances are below solar. Also, we compared the observed line profiles to synthetic spectra computed with variable accretion rates and vertical abundance distributions assuming diffusion steady-state. The inferred accretion rates vary from $\dot{M}=2 \times 10^{6}$ for calcium to $2 \times 10^{9} \mathrm{~g} \mathrm{~s}^{-1}$ for oxygen and indicate that the accretion flow is dominated by oxygen, silicon and iron while being deficient in carbon, magnesium and calcium. The lack of radial velocity variations between two measurement epochs suggests that GALEX J1931+0117 is probably not in a close binary and that the source of the accreted material resides in a debris disc.
\end{abstract}

Keywords: abundance, white dwarfs

PACS: $97.20 . \mathrm{Rp}$, 97.10.Tk

\section{INTRODUCTION}

GALEX J193156.8+011745 (GALEX J1931+0117, thereafter) is a hydrogen-rich white dwarf (Vennes, Kawka, \& Németh 2010) showing optical heavy-element lines and an infrared excess. The original low-resolution spectrum obtained with the New Technology Telescope (NTT) at La Silla Observatory showed a strong Mg II $\lambda 4481$ doublet and weaker silicon lines. Follow-up echelle spectroscopy obtained with the Very Large Telescope (VLT)-Kueyen enabled a detailed abundance study. The near-solar $( \pm 0.5$ dex $)$ abundances of oxygen, magnesium, silicon, calcium and iron bear the signature of an external supply of material accreting onto the surface of the white dwarf. Based on available data, Vennes et al. concluded that the supply may originate from a close, sub-stellar companion or from a cool debris disc.

The presence of heavy elements in hydrogen-rich white dwarfs has variously been interpreted as intrinsic to the star, or as extrinsic, i.e., supplied by the interstellar medium (Dupuis, Fontaine, \& Wesemael 1993), by a nearby companion as in post-common envelope systems (Debes 2006; Kawka et al. 2008), or by a debris disc (Zuckerman et al. 2003; Kilic et al. 2006; Farihi, Zuckerman, \& Becklin 2008). However, accretion from the interstellar medium is unlikely because of supply shortages (Farihi et al. 2010a). In the extrinsic scenarios, the elements are accreted and diffused in the atmosphere and envelope of the star (see Fontaine \& Michaud 1979; Koester 2009). An intrinsic, or inter- 
nal, reservoir of heavy elements is also possible, but in either scenario a self-consistent solution of the diffusion equation must explore the effect of radiative acceleration on trace elements (Chayer, Fontaine, \& Wesemael 1995; Chayer et al. 1995).

As a class, the polluted DA white dwarfs, or DAZs, are often defined by the detection of the Ca II H\&K doublet in optical spectra (Zuckerman et al. 2003; Koester et al. 2005). Exceptionally, $\mathrm{Mg}$ II $\lambda 4481$ is, so far, only detected in a handful of warm white dwarfs (but see Kawka et al. 2010) such as EG 102 (Holberg, Barstow, \& Green 1997), GALEX J1931+0117, and two warm white dwarfs from the Sloan Digital Sky Survey (SDSS) that show evidence of dusty and gaseous discs (Gänsicke et al. 2006; Gänsicke, Marsh, \& Southworth 2007). The presence of a large concentration of magnesium in the last two objects helped establish a strong link between heavy element pollution and dense circumstellar environments. Moreover, an infrared excess that cannot otherwise be explained by a cool companion, may be attributed to a dust ring as in the case of the DAZ white dwarf G29-38 (Graham et al. 1990).

We present new high-dispersion spectroscopic observations that help elucidate the nature of the peculiar abundance pattern in GALEX J1931+0117. We revise our abundance measurements and explore the effect of vertical abundance inhomogeneities.

\section{OPTICAL SPECTROSCOPY AND ABUNDANCES}

Following the identification of GALEX J1931+0117 as a DAZ white dwarf we obtained a series of echelle spectra $(R \approx 46000)$ using standard settings with the UV-Visual Echelle Spectrograph (UVES) attached to the VLT-Kueyen. The first series of spectra were obtained on UT 2009 Nov 12 (epoch 1, thereafter) with the dichroic \#2 and the "HER_5" and "BK7_5" filters on the blue and red arms, respectively. The blue spectrum was centred at $4370 \AA$ and covered the range $3757-4985 \AA$, and the red spectra were centred at $7600 \AA$ and covered the ranges 5698-7532 and 7660-9464 $\AA$. The first series of spectra were analysed by us (Vennes, Kawka, \& Németh 2010). The second series of spectra were obtained on UT 2010 Mar 15 (epoch 2, thereafter) with the dichroic \#1 and the "HER_5" and "SHP700" filters on the blue and red arms, respectively. The blue spectrum was centred at $3900 \AA$ and covered the range $3290-4518 \AA$, and the red spectra were centred at 5800 $\AA$ and covered the ranges $4788-5750$ and 5839-6808

The $\mathrm{H} \alpha$ radial velocity measured at both epochs are identical within errors. We adopted the average $v_{\mathrm{H} \alpha}=37.2 \pm 0.6 \mathrm{~km} \mathrm{~s}^{-1}$. The lack of radial velocity variations rules-out the presence of a close companion and supports the debris disc model. Figure 1 shows Si II lines with the velocity scale centred on $V_{\mathrm{H} \alpha}$. Stark shifts (González et al. 2002; Lesage 2009) are apparent in some lines. Figure 2 compares observed line spectra to the model line profiles that include or exclude Stark shifts. The Stark shifts result in a line asymmetry and a notable velocity shift. Details of the calculations are given by Vennes, Kawka, \& Németh (2011). Table 1 summarizes our new abundance measurements based on a critical review of atomic data (Vennes, Kawka, \& Németh 2011). 


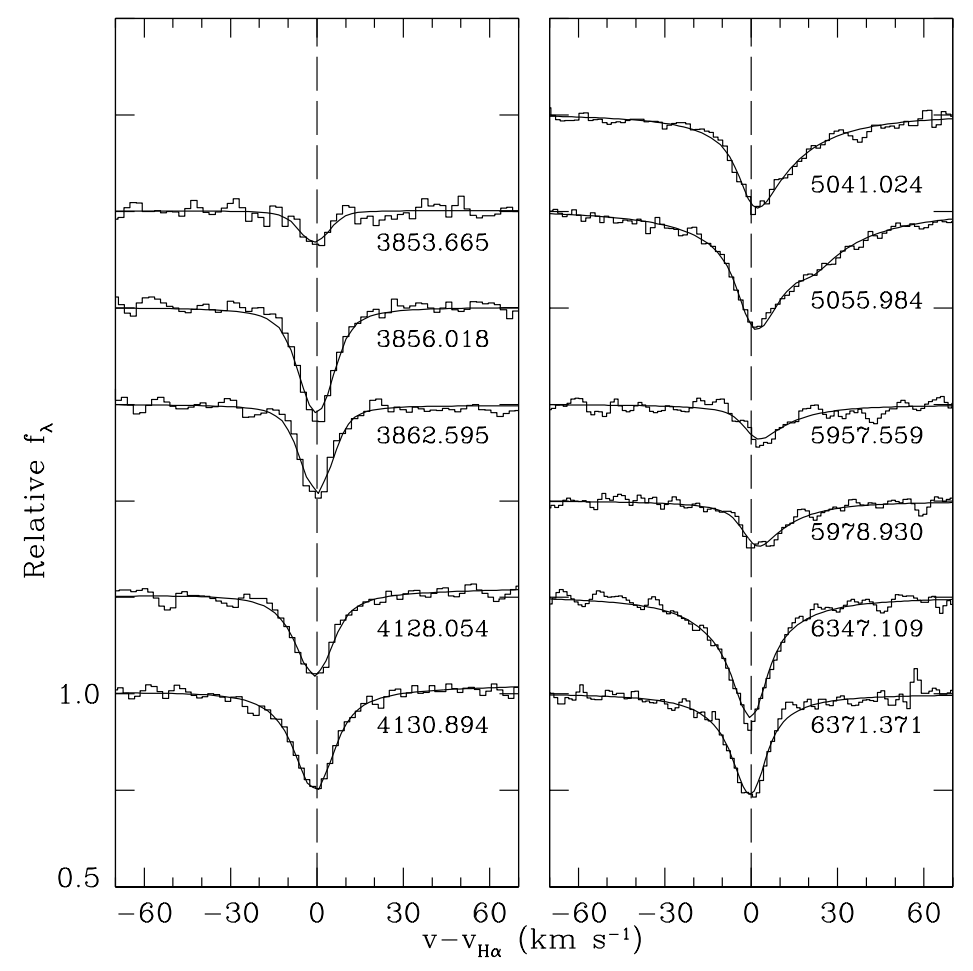

FIGURE 1. Set of eleven Si II lines obtained with the VLT/UVES. The line profile analysis reveals an abundance of silicon nearly a factor of two above solar. The Si II $\lambda \lambda 5041,5055,5957,5978$ lines show pressure (Stark) shifts in agreement with laboratory measurements.

\section{ACCRETION AND DIFFUSION IN THE ATMOSPHERE}

In the accretion/diffusion model the abundance at each layer is obtained by solving simultaneously the mass-continuity and diffusion equations. The mass-continuity equation for element " 2 " is

$$
\dot{M}_{2}=4 \pi R^{2}\left(A_{2} n_{2}\right) v_{1,2}
$$

where $R$ is the stellar radius, and $\dot{M}_{2}, A_{2}, n_{2}$, and $v_{1,2}$ are the mass accretion rate, the atomic weight, the number density, and the diffusion velocity of element "2", respectively. The diffusion velocity is given by the diffusion equation. Assuming diffusion steady-state the mass-continuity equation is solved for the abundance ratio

$$
c_{2} \equiv \frac{n_{2}}{n_{1}} \approx \dot{M}_{2} \frac{1}{4 \pi R^{2}} \frac{1}{A_{2}} \frac{\tau_{1,2}}{m},
$$

where $c_{2}<<1$ is the abundance of trace element "2" relative to the main constituent "1" assumed to be hydrogen and $\tau_{1,2}$ is the diffusion time scale of element "2" related to the diffusion velocity by

$$
\tau_{1,2}=\frac{m}{\rho v_{1,2}},
$$




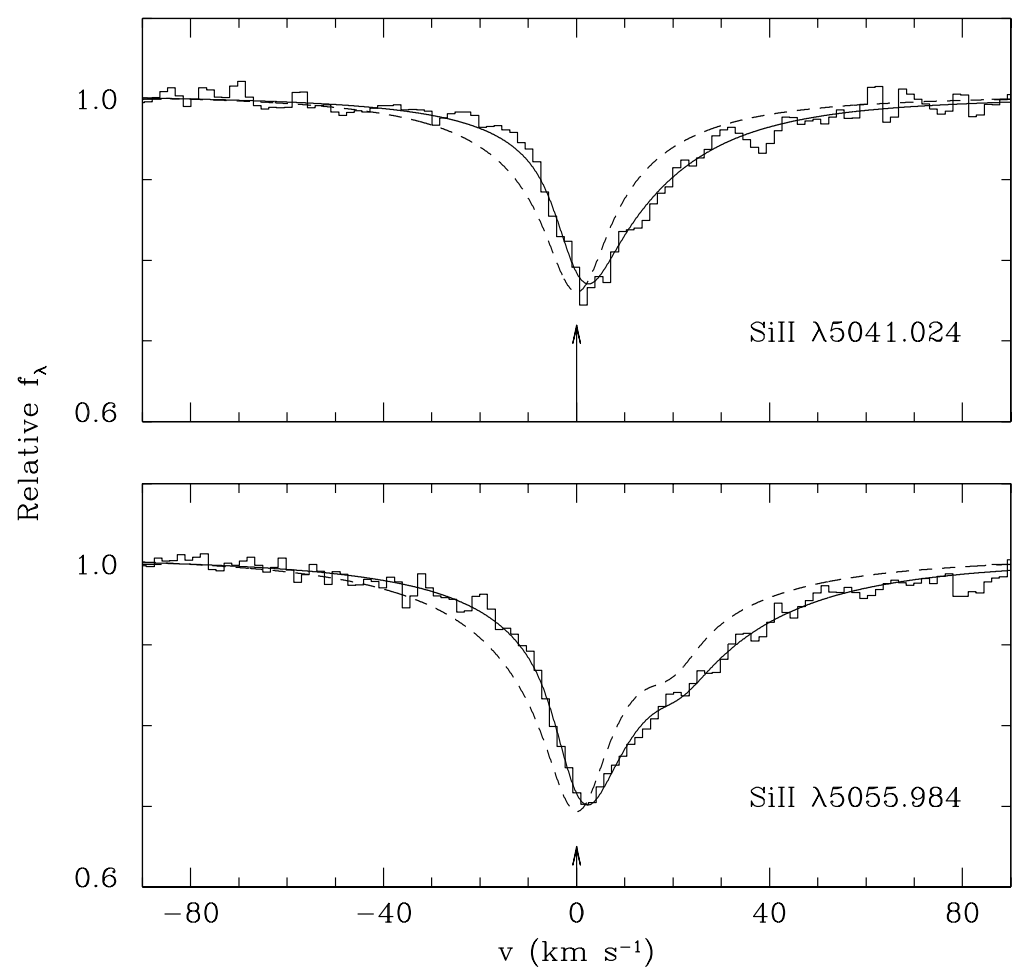

FIGURE 2. Details of SiII line profiles showing an asymmetry and a velocity shift. Model including pressure (Stark) shifts (full lines) are markedly superior to models excluding it (dashed lines).

where $m$ and $\rho$ are the total mass loading and density in the atmosphere. The selective radiation pressure and concentration gradients are negligible in the present case and the diffusion velocity is given by

$$
v_{1,2} \approx D_{1,2}\left(1-A_{2}\right) \frac{m_{p} g}{k T}
$$

where $D_{1,2}$ is the diffusion coefficient of element " 2 " which is a function of temperature, density and atomic charge. The diffusion coefficient for ionized species is given by Fontaine \& Michaud (1979) and includes a correction factor for thermal diffusion. Montmerle \& Michaud (1976) proposed to calculate the diffusion coefficient for neutral species based on the hard-sphere model:

$$
D_{1,2}(\mathrm{~h}-\mathrm{s})=1.36 \times 10^{19} \sqrt{\frac{T\left(A_{2}+1\right)}{A_{2}}} \frac{1}{\sigma_{0}^{2} n_{p}} \mathrm{~cm}^{2} \mathrm{~s}^{-1}
$$

where $T$ is the temperature in $\mathrm{K}, n_{p}$ is the proton density in $\mathrm{cm}^{-3}$, and $\sigma_{0}$ is the atomic radius (in $\AA$ ) taken as $0.91,0.65,1.72,1.46,2.23$, and 1.72 for $\mathrm{C}, \mathrm{O}, \mathrm{Mg}, \mathrm{Si}, \mathrm{Ca}$, and $\mathrm{Fe}$, respectively. However, Michaud, Martel, \& Ratel (1978) showed that the neutral-proton interaction is dominated by the induced electric dipole and that the hard-sphere model 
TABLE 1. Abundances and accretion rates in GALEX J1931+0117.

\begin{tabular}{ccccc}
\hline $\mathrm{X}$ & $n(\mathrm{X}) / n(\mathrm{H})$ & {$[\mathrm{X} / \mathrm{H}]^{\mathrm{a}}$} & $\log \dot{M}\left(\mathrm{~g} \mathrm{~s}^{-1}\right)$ & {$[\mathrm{X} / \mathrm{Si}]^{\mathrm{b}}$} \\
\hline $\mathrm{C}$ & $<7 \times 10^{-5}$ & $<-0.54$ & $<8.0$ & -0.8 \\
$\mathrm{O}$ & $2.4 \pm 0.3 \times 10^{-4}$ & -0.28 & $9.30 \pm 0.05$ & +0.1 \\
$\mathrm{Mg}$ & $3.8 \pm 0.6 \times 10^{-5}$ & +0.05 & $7.75 \pm 0.06$ & -0.5 \\
$\mathrm{Si}$ & $5.8 \pm 1.0 \times 10^{-5}$ & +0.25 & $8.25 \pm 0.07$ & $\ldots$ \\
$\mathrm{Ca}$ & $7.7 \pm 0.8 \times 10^{-7}$ & -0.42 & $6.35 \pm 0.04$ & -0.9 \\
$\mathrm{Fe}$ & $3.7 \pm 0.8 \times 10^{-5}$ & +0.12 & $8.50 \pm 0.09$ & +0.0 \\
\hline
\end{tabular}

${ }^{\mathrm{a}}[\mathrm{X} / \mathrm{H}] \equiv \log n(\mathrm{X}) / n(\mathrm{H})-\log n(\mathrm{X}) / n(\mathrm{H}) \odot$

$\mathrm{b}_{[\mathrm{X} / \mathrm{Si}]} \equiv \log n(\mathrm{X}) /\left.n(\mathrm{Si})\right|_{\text {flow }}-\log n(\mathrm{X}) / n(\mathrm{Si})_{\odot}$

may overestimate the diffusion coefficient by a large factor. The polarization diffusion coefficient is approximated by

$$
D_{1,2}(\mathrm{pol})=3.3 \times 10^{16} T \sqrt{\frac{A_{2}+1}{A_{2} \alpha_{D}}} \frac{1}{n_{p}} \mathrm{~cm}^{2} \mathrm{~s}^{-1}
$$

where $\alpha_{D}$ is the dipole polarizability (in $\AA^{3}$ ). We adopted averaged values of $1.68,0.86$, 10.6, 5.49, 23.6, and 8.95 for $\mathrm{C}, \mathrm{O}, \mathrm{Mg}, \mathrm{Si}, \mathrm{Ca}$, and Fe, respectively from the tabulation of P. Schwerdtfeger (http://ctcp.massey.ac.nz/dipole-polarizabilities). For example, at a reference temperature of $20 \times 10^{3} \mathrm{~K}$, the ratio of hard-sphere to polarization diffusion coefficients is $D_{1,2}(\mathrm{~h}-\mathrm{s}) / D_{1,2}(\mathrm{pol}) \approx 3$ for $\mathrm{Mg}, \mathrm{Si}, \mathrm{Ca}$, and $\mathrm{Fe}, \approx 5$ for $\mathrm{C}$, and $\approx 6$ for $\mathrm{O}$. The diffusion coefficient averaged over ionization species is given by:

$$
D_{1,2}=\sum_{i} x_{i} D_{1,2, i}
$$

where $x_{i}=n_{2, i} / n_{2}$ are the ionization fractions for element "2" and $D_{1,2, i}$ are the corresponding diffusion coefficients.

Figure 3 shows the predicted abundance profiles of $\mathrm{O}, \mathrm{Mg}, \mathrm{Si}$, and $\mathrm{Ca}$ in the atmosphere of GALEX J1931+0117 calculated using the procedure described above. The accretion rates were set at the best-fitting values. The effect of neutral species on the profiles of $\mathrm{Mg}, \mathrm{Si}$, and $\mathrm{Ca}$ is negligible because of their relatively high ionization fractions. On the other hand, the predicted oxygen abundance profile in the line forming region is dominated by diffusion of neutral oxygen atoms. Employing the hard-sphere diffusion coefficient for neutral oxygen leads to an overestimation of the diffusion velocity and an underestimation of the abundance by up to a factor of 4 relative to more realistic calculations that employ the polarization diffusion coefficient as determined by Michaud, Martel, \& Ratel (1978).

Table 1 lists the accretion rates $\dot{M}$ for each element measured by fitting spectral syntheses based on calculated abundance profiles to the observed line spectra. The inferred abundances in the accretion flow reveal a relatively high abundance of oxygen, silicon, and iron relative to carbon, magnesium and calcium. 


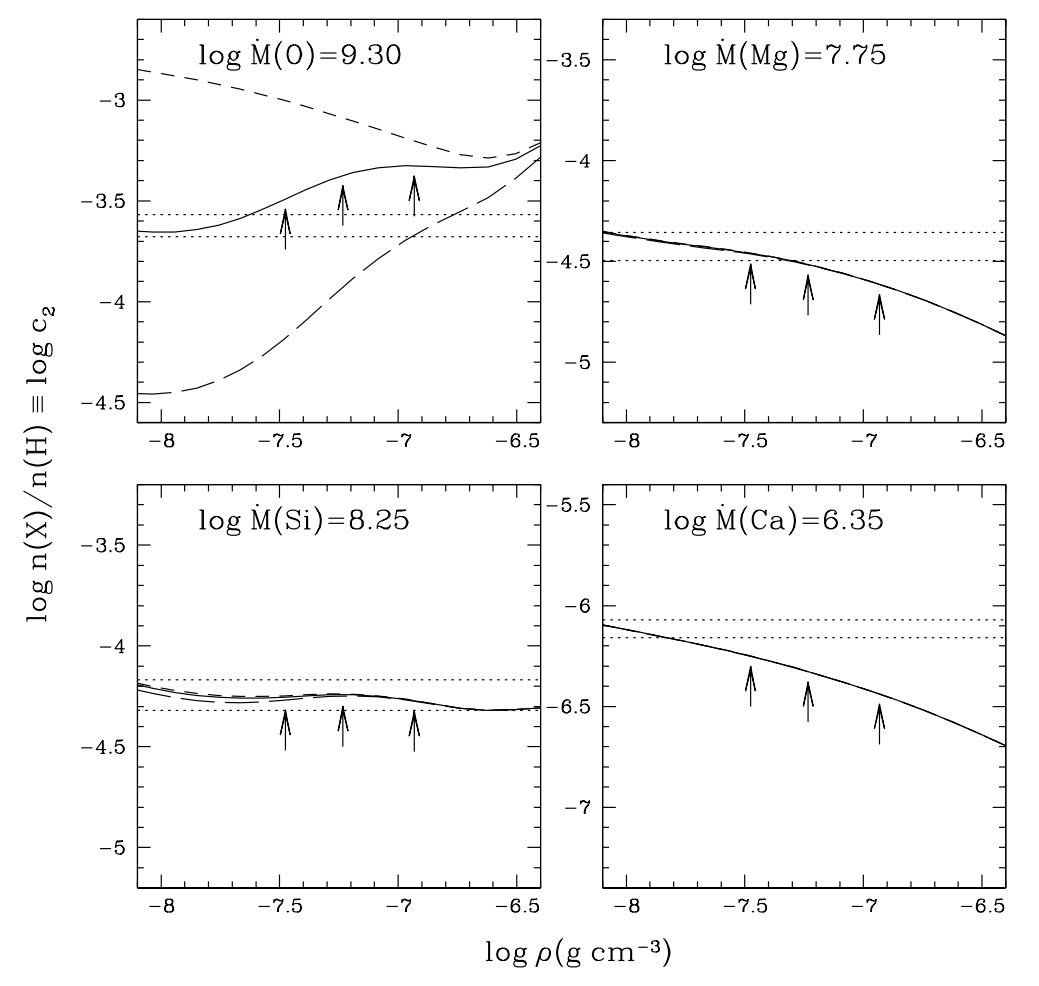

FIGURE 3. Abundance profiles in the line forming region predicted by the accretion-diffusion model. The accretion rates that fit the observed $\mathrm{O}, \mathrm{Mg}, \mathrm{Si}$, and $\mathrm{Ca}$ line spectra are given in $\mathrm{g} \mathrm{s}^{-1}$. The abundances measured using homogeneous model atmospheres are shown with dotted horizontal lines. Diffusion profiles excluding neutral species (short dashed lines) overestimate the abundances in the line forming region relative to models including neutral species and realistic polarization diffusion coefficients (full lines), while models using hard-sphere diffusion coefficients (long dashed lines) underestimate the abundances. Mass densities corresponding to optical depth unity at $\lambda=7400$ (leftmost), 4700, and $1260 \AA$ (rightmost) are marked with vertical arrows.

\section{SPECTRAL ENERGY DISTRIBUTION}

A lack of radial velocity variations suggests that the source of the accreted material is a circumstellar debris disc. Simulated infrared spectral energy distributions for GALEX J1931+0117 including a spectral synthesis of the white dwarf added to a warm disc (Fig. 4) show that the observed distribution (Vennes, Kawka, \& Németh 2010) can be matched by a warm disc $(900 \mathrm{~K})$ or a hot disc $(1650 \mathrm{~K})$ near sublimation temperature. The inferred dust temperature must be below sublimation temperatures (see Lodders 2003 ) for the main constituents such as calcium ( $1659 \mathrm{~K})$, or silicon and magnesium $(1354 \mathrm{~K})$. The corresponding disc sizes are $90 \Omega_{W D}$ for the hot disc, and, compensating for its reduced emissivity per unit area, $2200 \Omega_{W D}$ for the warm disc, where $\Omega_{W D}$ is the solid angle subtended by the white dwarf itself. The observed spectral energy distribution extends to the 2MASS measurements in the infrared and measurements at longer wavelengths are required to further constrain the model.

A comparison with other warm DAZ white dwarfs supports these conjectures. The 


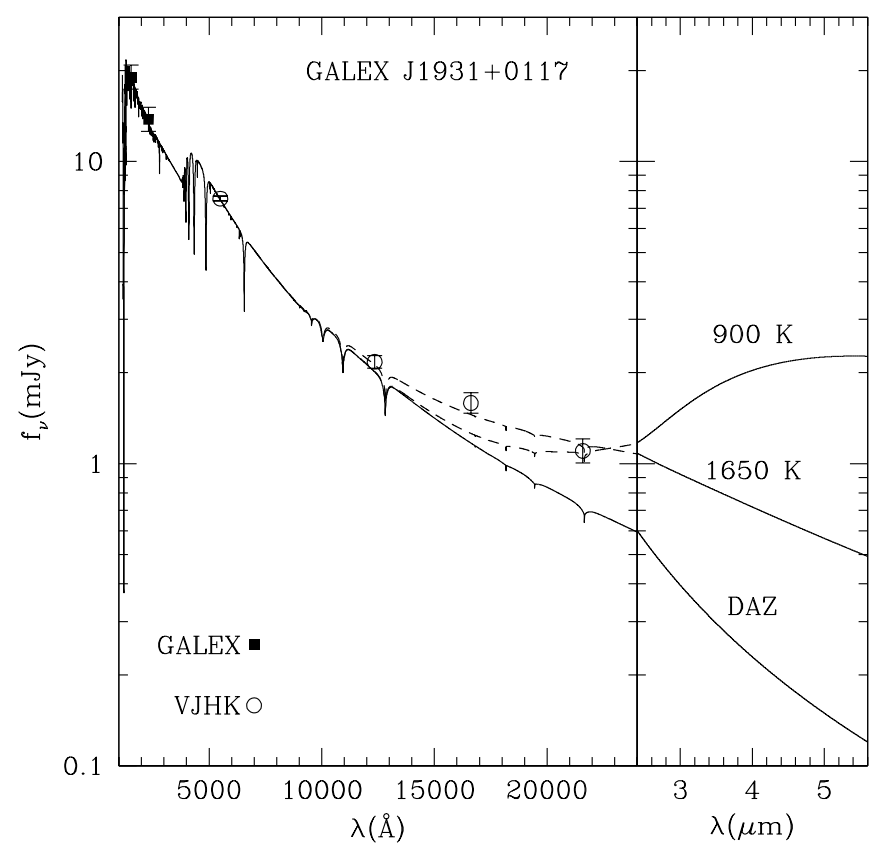

FIGURE 4. Predicted spectral energy distribution of GALEX J1931+0117 (full lines) extending beyond the JHK photometry and including the emergent flux of a hot white dwarf (DAZ) added to black-body emission from a disc at 900 or $1650 \mathrm{~K}$. The white dwarf dominates the ultraviolet region (GALEX $N_{\mathrm{UV}}$ and $F_{\mathrm{UV}}$ ) while the disc is predicted to dominate the infrared region.

DAZ PG1015+161 (Koester \& Wilken 2006) is similar to GALEX J1931+0117, and, based on JHK and Spitzer observations, Jura, Farihi, \& Zuckerman (2007) inferred the presence of a disc with an inner temperature ranging from 800 to $1000 \mathrm{~K}$ and emitting area from 700 to $1200 \Omega_{W D}$. In a similar case, Farihi, Jura, \& Zuckerman (2009) inferred the presence of a disc with a blackbody temperature of 1500K around PG1457-086. The great diversity in disc emissivity is illustrated by the case of HS0047+1903. Despite having a similar calcium abundance, no evidence of a disc is found in JHK and Spitzer observations (Farihi et al. 2010b).

\section{CONCLUSIONS}

We presented a model atmosphere analysis of the high-metallicity white dwarf GALEX J1931+0117. The abundance pattern obtained using homogeneous model atmospheres shows that magnesium, silicon, and iron are near or above solar abundances, while carbon, oxygen and calcium are below solar abundances. However, a line profile analysis performed using vertical abundance distributions obtained by solving the steady-state diffusion equation shows that the accretion flow is rich in oxygen, silicon, and iron while it is depleted in other elements. Although the oxygen abundance is below solar in the line forming region, it must be supplied in larger quantity because 
of its short diffusion time-scale relative to other elements.

The effect of pressure shifts are apparent in several strong silicon lines. This effect predicted by Hammond (1989) and Kršljanin \& Dimitrijević (1992) also impacts radial velocity measurements. A lack of radial velocity variations between two epochs that are 123 days apart rules out a close binary scenario for the origin of the accreted material. We are left with the possibility that the infrared excess belongs to a warm disc of debris material that accretes onto the white dwarf surface.

In conclusion, our analysis of the abundance pattern in GALEX J1931+0117 and the absence of a close companion, as well as a comparison with similar cases (e.g., DAZ PG1015+161) support the likely presence of a dusty disc around GALEX J1931+0117.

\section{ACKNOWLEDGMENTS}

This research is supported by GA AV grant numbers IAA301630901 and IAA300030908, respectively, and by GA ČR grant number P209/10/0967.

\section{REFERENCES}

Chayer, P., Fontaine, G., Wesemael, F., 1995, ApJS, 99, 189

Chayer, P., Vennes, S., Pradhan, A. K., Thejll, P., Beauchamp, A., Fontaine, G., Wesemael, F., 1995, ApJ, 454, 429

Debes, J. H., 2006, ApJ, 652, 636

Dupuis, J., Fontaine, G., Wesemael, F., 1993, ApJS, 87, 345

Farihi, J., Barstow, M. A., Redfield, S., Dufour, P., Hambly, N. C., 2010a, MNRAS, 404, 2123

Farihi, J., Jura, M., Lee, J.-E., Zuckerman, B., 2010b, ApJ, 714, 1386

Farihi, J., Jura, M., Zuckerman, B., 2009, ApJ, 694, 805

Farihi, J., Zuckerman, B., Becklin, E. E., 2008, ApJ, 674, 431

Fontaine, G., Michaud, G., 1979, ApJ, 231, 826

Gänsicke, B. T., Marsh, T. R., Southworth, J., 2007, MNRAS, 380, L35

Gänsicke, B. T., Marsh, T. R., Southworth, J., Rebassa-Mansergas, A., 2006, Sci, 314, 1908

González, V. R., Aparicio, J. A., del Val, J. A., Mar S., 2002, JPhB, 35, 3557

Graham, J. R., Matthews, K., Neugebauer, G., Soifer, B. T., 1990, ApJ, 357, 216

Hammond, G. L., 1989, LNP, 328, 346

Holberg, J. B., Barstow, M. A., Green, E. M., 1997, ApJ, 474, L127

Jura, M., Farihi, J., Zuckerman, B., 2007, ApJ, 663, 1285

Kawka, A., Vennes, S., Dupuis, J., Chayer, P., Lanz, T., 2008, ApJ, 675, 1518

Kawka, A., Vennes, S., Dinnbier, F., Cibulková, H., \& Németh, P. 2010, these proceedings

Kilic, M., von Hippel, T., Leggett, S. K., Winget, D. E., 2006, ApJ, 646, 474

Koester, D., 2009, A\&A, 498, 517

Koester, D., Rollenhagen, K., Napiwotzki, R., Voss, B., Christlieb, N., Homeier, D., Reimers, D., 2005, A\&A, 432, 1025

Koester, D., Wilken, D., 2006, A\&A, 453, 1051

Kršljanin, V., Dimitrijević, M. S., 1992, LNP, 401, 371

Lesage, A., 2009, NewAR, 52, 471

Lodders, K., 2003, ApJ, 591, 1220

Michaud, G., Martel, A., \& Ratel, A. 1978, ApJ, 226, 483

Montmerle, T., Michaud, G., 1976, ApJS, 31, 489

Vennes, S., Kawka, A., \& Németh, P., 2010, MNRAS, 404, L40

Vennes, S., Kawka, A., \& Németh, P., 2011, MNRAS, submitted

Zuckerman, B., Koester, D., Reid, I. N., Hünsch, M., 2003, ApJ, 596, 477 